\title{
Melanoma Diagnosis: The Importance of Histopathological Report
}

\author{
Alessandra Filosa ${ }^{a}$ Giorgio Filosab \\ a Pathological Anatomy Unit, AV3, Macerata Hospital, Macerata, Italy; ${ }^{b}$ Dermatology Unit, \\ AV2, Carlo Ubani Hospital, Jesi, Italy
}

Keywords
Melanoma $\cdot$ Histopathology $\cdot$ Consensus diagnosis

The demand for histopathological diagnosis of pigmented lesions has increased steadily over the last 20 years, partly due to the incidence of melanoma and partly because of increased awareness and referrals from general practitioners. This makes a unified and systematic approach to melanoma histopathology even more important. The histopathological diagnosis of melanocytic lesions is one of the greatest challenges for pathologists. The recognition of a melanocytic tumor as a melanoma is not based on the search of single, objective, and easily reproducible morphological diagnostic features but, instead, it stems from a constellation of diagnostic criteria whose implementation, meaning, and relative weight may vary considerably from one case to another [1]. In the pathological reporting of a primary malignant melanoma, the desirable features include: characteristics of the melanoma, including subtype; whether the lesion has been completely excised; and evaluation of prognostic indicators. This will allow critical clinical decisions in order to avoid local recurrences and possible sources of metastases [1,2]. There is no single histological criterion that definitely separates benign from malignant melanocytic lesions. Every histological feature that has been reported in melanoma has also been found in benign nevi. Moreover each type of benign nevus may simulate malignant melanoma and vice versa. The histopathological characteristics must be evaluated in conjunction with clinical and macroscopic data, and the histologic features have to be assessed in the clinical context. This stresses the importance of the clinician providing all relevant data in the pathology request form including age, sex, site, pregnancy, history of previous melanomas or nevi in the site of the current lesion or elsewhere, and recent change in the lesion. Accurate diagnosis may sometimes require a close consultation between the 


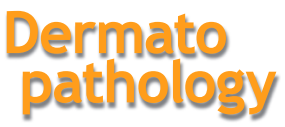

Table 1. The 2006 WHO

classification of melanoma
Dermatopathology 2018;5:41-43

(c) 2018 The Author(s). Published by S. Karger AG, Basel www.karger.com/dpa

Filosa and Filosa: Melanoma Diagnosis: The Importance of Histopathological Report

Superficial spreading melanoma

Nodular melanoma

Lentigo maligna

Acral lentiginous melanoma

Desmoplastic and desmoplastic neurotropic melanoma

Melanoma arising from blue nevus

Melanoma arising in a giant congenital nevus

Melanoma of childhood

Nevoid melanoma

Persistent melanoma

Ulceration

Mitotic rate

Regression

Lymphovascular invasion

Perineural invasion

Breslow thickness

Satellitosis

Status of the surgical margins
Table 3. Optional

histopathological features in the pathology report
Histological subtype

Cell type

Amount of pigmentation

Clark level

Tumor growth phase

Tumor infiltrating lymphocytes

Associated nevus

pT according to AJCC ed. 8

dermatologist and the pathologist $[3,4]$. The historical classification into lentigo maligna melanoma, superficial spreading melanoma, nodular melanoma, and acral lentiginous melanoma is still an acceptable start point $[5,6]$. The WHO 2006 classification recognizes additional subtypes of melanoma [2], but is still incomplete (Table 1). The histological diagnosis of melanocytic lesions requires assessment of architectural and cytological features. Histological features that favor a diagnosis of melanoma include asymmetry, ulceration, cytological atypia, pagetoid involvement of the epidermis, lack of maturation, and dermal mitosis with deep and atypical ones. Optimal evaluation of any melanocytic lesion requires complete excision that incorporates the full lesion instead of incorporating the full thickness of the involved lesion removed intact $[4,5]$. Because of the lack of objective and reproducible diagnostic criteria, ancillary techniques such as immunohistochemistry have been increasingly implemented in routine practice. However, immunohistochemistry must be always evaluated in the morphological context, because any single immunostain is not able to give clear-cut information for a differential diagnosis between nevus and melanoma, because there are still no reliable markers that are both highly sensitive and specific for melanoma diagnosis [6]. Moreover, molecular techniques are being increasingly proposed with the aim of looking for specific pathways toward melanoma genesis $[5,6]$, but histopathology remains the major source of the most reproducible tool for diagnosis and prognosis in melanoma. Mandatory 
histopathological parameters are listed in Table 2. Optional parameters of the histopathological report are listed in Table 3. When the histopathological report is rendered, the clinicians review the case considering the clinical and dermoscopic findings instead of clinicaldermatoscopic pictures. A final consensus diagnosis should be reached in light of the case discussion. To conclude, the pathologist should provide the dermatologist with a report containing sufficient information to allow an evidence-based management plan to be made for the patient and to determine accurate prognosis.

\section{Statement of Ethics}

The authors have no ethical conflicts to disclose.

\section{Disclosure Statement}

The authors have no conflicts of interest to disclose.

\section{References}

1 Thompson JF, Morton DL, Kroon BBR (eds): Textbook of Melanoma: Pathology, Diagnosis and Management. London, Martin Dunitz, 2003.

2 De Vries E, Bray F, Coebergh GW, Cerroni L, Ruiter DJ, Elder DE: Malignant melanoma: Introduction; in Le Boit PE, Burg G, Weedon D, Sarasin A (eds): WHO Classification of Tumours - Pathology and Genetics of Skin Tumours. Lyon, IARC Press, 2006, pp 52-65.

3 Abbas 0, Miller DD, Bhawan J: Cutaneous malignant melanoma: update on diagnostic and prognostic biomarkers. Am J Dermatopathol 2014;36:363-379.

4 Clark WH Jr, From L, Bernardino EA, Mihm MA: The histogenesis and biologic behavior of primary malignant melanoma of the skin. Cancer Res 1969:29:705-727.

5 Patravala S. Malay A, Greskovich C, Stuart L, Parker D, Swerlick R: Discordance of histopathologic parameters in cutaneous melanoma: clinical implication. J Am Acad Dermatol 2016;74:75-80.

6 Prieto VG, Shea CR: Immunohistochemistry of melanocytic proliferation. Arch Pathol Lab Med 2011;135:853859. 